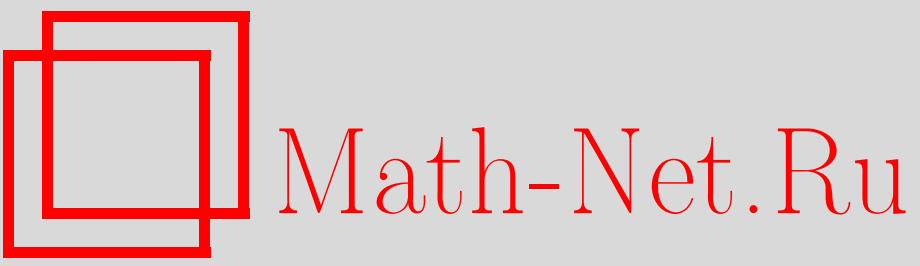

А. Б. Шидловский, О критериях линейной и алгебраической зависимости значений $E$-функций в алгебраических точках, УМH, 1996, том 51, выпуск 2, 179-180

DOI: https://doi.org/10.4213/rm965

Использование Общероссийского математического портала Math-Net.Ru подразумевает, что вы прочитали и согласны с пользовательским соглашением

http://www.mathnet.ru/rus/agreement

Параметры загрузки:

IP: 54.210 .77 .194

26 апреля 2023 г., 16:44:26 


\title{
О КРИТЕРИЯХ ЛИНЕЙНОЙ И АЛГЕБРАИЧЕСКОЙ ЗАВИСИМОСТИ ЗНАЧЕНИЙ Е-ФУНКЦИЙ В АЛГЕБРАИЧЕСКИХ ТОЧКАХ
}

\author{
А. Б. Шидловский
}

Формулируются теоремы о необходимых и достаточных условиях линейной и алгебраической зависимости значений $E$-функций в алгебраических точках.

С историей вопроса и многими результатами об арифметических свойствах значений $E$-функций можно ознакомиться по книге [1]. В статье [2] доказан ряд теорем и высказано несколько предположений о линейной независимости значений $E$-фрункций.

Пусть в дальнейшем совокупность $E$-функций

$$
f_{1}(z), \ldots, f_{m}(z)
$$

будет составлять решение системы линейных однородных дифференциальных уравнений

$$
y_{k}^{\prime}=\sum_{i=1}^{m} Q_{k, i} y_{i}, \quad k=1, \ldots, m, \quad Q_{k, i} \in \mathbb{C}(z), \quad m \geqslant 2
$$

или системы дифференциальных уравнений

$$
y_{k}^{\prime}=Q_{k, 0}+\sum_{i=1}^{m} Q_{k, i} y_{i}, \quad k=1, \ldots, m, \quad Q_{k, i} \in \mathbb{C}(z), \quad m \geqslant 1,
$$

которая может быть и однородной. Пусть $T(z) \in \mathbb{C}[z]$ и является общим наименьшим знаменателем всех функций $Q_{k}, i$ в системе $(2)$ или $(3)$, а $\xi \in \mathbb{A}, \xi T(\xi) \neq 0$, где $\mathbb{A}$ - поле всех алгебраических чисел над $\mathbb{Q}$. Пусть также $\mathbb{K}$ обозначает любое фиксированное алгебраическое поле над $\mathbb{Q}$, содержашее коэффициенты степенных рядов по степеням $z$ всех $E$-функций (1) и число $\xi$.

Теорема 1. Пусть Е-функиии (1) составляют решение системы (2),

$$
f_{i_{1}}(z), \ldots, f_{i_{t}}(z), \quad 1 \leqslant t \leqslant m,
$$

- любая подсовокупность функций (1), а $\xi \in \mathbb{A}, \xi T(\xi) \neq 0$.

Тогда числа

$$
f_{i_{1}}(\xi), \ldots, f_{i_{t}}(\xi)
$$

линейно зависимь над полем $\mathbb{K}$ в том и только в том случае, когда существует неприводимый однородный многочлен $P=P\left(z, z_{1}, \ldots, z_{m}\right) \in \mathbb{K}\left[z, z_{1}, \ldots, z_{m}\right]$ такой, что

$$
P\left(z, f_{1}(z), \ldots, f_{m}(z)\right)=0
$$

$$
P\left(\xi, z_{1}, \ldots, z_{m}\right)=\left(\sum_{s=1}^{t} a_{s} z_{i_{s}}\right) P_{1}\left(z_{1}, \ldots, z_{m}\right),
$$

¿de

$$
\begin{gathered}
a_{1}, \ldots, a_{t} \in \mathbb{K}, \quad \sum_{s=1}^{t}\left|a_{s}\right|>0, \quad \sum_{s=1}^{t} a_{s} f_{i_{s}}(\xi)=0, \\
P_{1}\left(z_{1}, \ldots, z_{m}\right) \in \mathbb{K}\left[z_{1}, \ldots, z_{m}\right] .
\end{gathered}
$$

Работа выполнена при частичной финансовой поддержке Международного научного фонда (грант № MHS000). 
ЗАмЕЧАнИЕ. У тверждение теоремы 1 сохранится, если в ее формулировке заменить поле $\mathbb{K}$ на поле $\mathbb{A}$.

Рассматривая совокупность $E$-функций (1), составляющую решение системы (3), заменяя в теореме 1 число $m$ на $m+1$ и полагая $f_{m+1}(z) \equiv 1$, получим неоднородный аналог теоремы 1 о линейной зависимости чисел $f_{i_{1}}(\xi), \ldots, f_{i_{t}}(\xi)$ и 1.

В работе [4] (теорема 3 ) доказан критерий алгебраической зависимости значений подсовокупности функций (1). Такого типа критерий выполняется в более общем случае.

Теорема 2. Пусть Е-функции (1) составляют решение системы (3) (системы (2)), функции (4) - любая подсовокупность функций (1), а $\xi \in \mathbb{A}, \xi T(\xi) \neq 0$.

Тогда числа (5) алгебраически зависимы (однородно алгебраически зависимы) в том и только в том случае, когда существует неприводимый (однородный неприводимый) многочлен

$$
P=P\left(z, z_{1}, \ldots, z_{m}\right) \in \mathbb{A}\left[z, z_{1}, \ldots, z_{m}\right]
$$

удовлетворяющий условию (6), такой, что

$$
\begin{gathered}
P\left(\xi, z_{1}, \ldots, z_{m}\right)=P_{0}\left(z_{i_{1}}, \ldots, z_{i_{t}}\right) P_{1}\left(z_{1}, \ldots, z_{m}\right), \\
P_{0}=P_{0}\left(z_{i_{1}}, \ldots, z_{i_{t}}\right) \in \mathbb{A}\left[z_{i_{1}}, \ldots, z_{i_{t}}\right], \quad P_{0}\left(f_{i_{1}}(\xi), \ldots, f_{i_{t}}(\xi)\right)=0,
\end{gathered}
$$

где $P_{0}$ - неприводимый многочлен, а $P_{1}\left(z_{1}, \ldots, z_{m}\right) \in \mathbb{A}\left[z_{1}, \ldots, z_{m}\right]$.

Применение теорем 1 и 2 к конкретным функциям, вообще говоря, затруднительно, так как в них утверждается только существование многочлена $P$ с указанными свойствами, но не указывается метод его нахождения. Поэтому представляет интерес устанавливать достаточные условия для доказательства линейной и алгебраической независимости значений функций (1), которые было бы возможно применять к каким-либо конкретным подклассам $E$-функций. Наиболее простым оказывается случай, когда $l=m-1$. В этом случае из теоремы 2 получается следующий результат.

Теорема 3. Пусть Е-функции (1) составляют решение системы (3) (системы (2)), степень трансцендентности (однородной трансцендентности) функций (1) над $\mathbb{C}(z)$ равна $m-1$, функции (4) - любая подсовокупность функций (1), $P$ - неприводимьй многочлен (однородный многочлен) (7) такой, что выполняется условие (6), а $\xi \in \mathbb{A}$, $\xi T(\xi) \neq 0$.

Тогда если в разложсении многочлена $P\left(\xi, z_{1}, \ldots, z_{m}\right)$ на неприводимые множители каждый такой множитель содержит хотя бъ одну из переменных $z_{i_{t+1}}, \ldots, z_{i_{m}}$, то числа (5) алгебраически (однородно алгебраически) независимь.

Аналогичное следствие получается и из теоремы 1 для линейной независимости чисел (5). При этом надо накладывать ограничения только на линейные множители многочлена $P$ (см. [3]).

Нетрудно привести примеры приложений теоремы 3 к конкретным функциям (см. [3])

\section{СПИСОК ЛИТЕРАТУРЫ}

[1] Шидловский А. Б. Трансцендентные числа. М.: Наука, 1987. [2] Шидловский А. Б. // Матем. заметки. 1994. Т. 55. № 2. С. 174-185. [3] Шидловский А. Б. // Матем. заметки. 1995. Т. 58. № 5. С. 794-797. [4] Шидловский А. Б. // Труды МИАН. 1994. Т. 207. С. 369-376.

Московский государственный

Принято редколлегией университет им. М. В. Ломоносова 